\title{
Heat-kernel approach for scattering
}

\author{
Wen-Du Li ${ }^{1}$, Wu-Sheng Dai ${ }^{1,2, a}$ \\ ${ }^{1}$ Department of Physics, Tianjin University, Tianjin 300072, People's Republic of China \\ 2 LiuHui Center for Applied Mathematics, Nankai University and Tianjin University, Tianjin 300072, People's Republic of China
}

Received: 31 March 2015 / Accepted: 8 June 2015 / Published online: 27 June 2015

(C) The Author(s) 2015. This article is published with open access at Springerlink.com

\begin{abstract}
An approach for solving scattering problems, based on two quantum field theory methods, the heat-kernel method and the scattering spectral method, is constructed. This approach converts a method of calculating heat kernels into a method of solving scattering problems. This allows us to establish a method of scattering problems from a method of heat kernels. As an application, we construct an approach for solving scattering problems based on the covariant perturbation theory of heat-kernel expansions. In order to apply the heat-kernel method to scattering problems, we first calculate the off-diagonal heat-kernel expansion in the frame of covariant perturbation theory. Moreover, as an alternative application of the relation between heat kernels and partialwave phase shifts presented in this paper, we give an example of how to calculate a global heat kernel from a known scattering phase shift.
\end{abstract}

\section{Introduction}

In this paper, based on two quantum field theory methods, heat-kernel method [1] and scattering spectral method [2], we present a new approach to solve scattering problems. This approach is a series of approaches for scatterings rather than a single approach. Concretely, our key result is an explicit relation between partial-wave scattering phase shifts and heat kernels. By this result, each method of calculating heat kernels leads to an approach of calculating phase shifts; or, in other words, the approach converts a method of solving heat kernels into a method of solving scattering problems. Many methods for scattering problems can be constructed by this approach, since the heat-kernel theory is well studied in both mathematics and physics and there are many mature methods for the calculation of heat kernels.

Phase shift. All information of an elastic scattering process is embedded in a scattering phase shift. This can be seen by

\footnotetext{
a e-mail: daiwusheng@tju.edu.cn
}

directly observing the asymptotic solution of the radial wave equation. For spherically symmetric cases, the asymptotic solution of the free radial wave equation, $\left[-\frac{1}{r^{2}} \frac{\mathrm{d}}{\mathrm{d} r}\left(r^{2} \frac{\mathrm{d}}{\mathrm{d} r}\right)+\right.$ $\left.\frac{l(l+1)}{r^{2}}\right] R_{l}=k^{2} R_{l}$, is $R_{l}(r) \stackrel{r \rightarrow \infty}{=}(1 / k r) \sin (k r-l \pi / 2)$, and the asymptotic solution of the radial wave equation with a potential, $\left[-\frac{1}{r^{2}} \frac{\mathrm{d}}{\mathrm{d} r}\left(r^{2} \frac{\mathrm{d}}{\mathrm{d} r}\right)+\frac{l(l+1)}{r^{2}}+V(r)\right] R_{l}=k^{2} R_{l}$, is

$R_{l}(r) \stackrel{r \rightarrow \infty}{=} \frac{1}{k r} \sin \left[k r-\frac{l \pi}{2}+\delta_{l}(k)\right]$.

This defines the partial-wave phase shift $\delta_{l}(k)$, which is the only effect on the radial wave function at asymptotic distances [3]. Therefore, all we need to do in solving a scattering problem is to solve for the phase shift $\delta_{l}(k)$.

Heat kernel. The information embedded in an operator $D$ can be extracted from a heat kernel $K\left(t ; \mathbf{r}, \mathbf{r}^{\prime}\right)$, which is the Green function of the initial-value problem of the heat-type equation $\left(\partial_{t}+D\right) \phi=0$, determined by [1]

$\left(\partial_{t}+D\right) K\left(t ; \mathbf{r}, \mathbf{r}^{\prime}\right)=0$, with $K\left(0 ; \mathbf{r}, \mathbf{r}^{\prime}\right)=\delta\left(\mathbf{r}-\mathbf{r}^{\prime}\right)$.

The global heat kernel $K(t)$ is the trace of the local heat kernel $K\left(t ; \mathbf{r}, \mathbf{r}^{\prime}\right): K(t)=\int d \mathbf{r} K(t ; \mathbf{r}, \mathbf{r})=\sum_{n, l} e^{-\lambda_{n l} t}$, where $\lambda_{n l}$ is the eigenvalue of the operator $D$.

The main aim of the present paper is to seek a relation between the partial-wave phase shift $\delta_{l}(k)$ and the heat kernel $K\left(t ; \mathbf{r}, \mathbf{r}^{\prime}\right)$. By this relation, we can explicitly express a partial-wave phase shift by a given heat kernel. There are many studies on the approximate calculation of heat kernels [1,4-13] and each approximate method of heat kernels gives us an approximate method for calculating partial-wave phase shifts.

The present work is based on our preceding work given in Ref. [14], which reveals a relation between two quantum field theory methods, the heat-kernel method [1] and the scattering 
spectral method [2]. In Ref. [14], using the relation between spectral counting functions and heat kernels given by Ref. [15] and the relation between phase shifts and state densities given by Ref. [2], we provide a relation between the global heat kernel and the total scattering phase shift (the total scattering phase shift is the summation of all partial-wave phase shifts, $\left.\delta(k)=\sum_{l}(2 l+1) \delta_{l}(k)\right)$.

Nevertheless, the result given by Ref. [14]—-the relation between total scattering phase shifts and heat kernels - can hardly be applied to scattering problems, since the total phase shift has no clear physical meaning.

To apply the heat-kernel method to scattering problems, we in fact need a relation between partial-wave phase shifts (rather than total phase shifts) and heat kernels. In the present paper, we find such a relation. This relation allows us to express a partial-wave phase shift by a known heat kernel. Then all physical quantities of a scattering process, such as scattering amplitudes and cross sections, can be expressed by a heat kernel.

To find the relation between partial-wave phase shifts and heat kernels, we will first prove a relation between heat kernels and partial-wave heat kernels. The heat kernel $K\left(t ; \mathbf{r}, \mathbf{r}^{\prime}\right)$ is the Green function of initial-value problem of the heat equation (2) with the operator $D=-\nabla^{2}+V(r)$ and the partial-wave heat kernel $K_{l}\left(t ; r, r^{\prime}\right)$ is the Green function of initial-value problem of the heat equation (2) with the radial operator $D_{l}=-\frac{1}{r^{2}} \frac{\mathrm{d}}{\mathrm{d} r}\left(r^{2} \frac{\mathrm{d}}{\mathrm{d} r}\right)+\frac{l(l+1)}{r^{2}}+V(r)$. By this relation, we can calculate a partial-wave heat kernel $K_{l}\left(t ; r, r^{\prime}\right)$ from a heat kernel $K\left(t ; \mathbf{r}, \mathbf{r}^{\prime}\right)$ directly.

The main aim of this paper is to explicitly express the partial-wave phase shift by a given heat kernel. As mentioned above, by our result, each method of calculating heat kernels can be converted to a method of calculating scattering problems.

In order to calculate a scattering phase shift from a heat kernel, we need off-diagonal heat kernels (i.e., heat kernels). For this purpose, in the following, we first calculate an offdiagonal heat-kernel expansion in the frame of the covariant perturbation theory. It should be pointed out that many methods on the calculation of diagonal heat-kernel expansions in literature can be directly apply to the calculation of off-diagonal heat kernels.

A method for calculating scattering phase shifts based on the covariant perturbation theory in the heat kernel theory is established as an example of our approach.

Furthermore, we compare the scattering method established in this paper, which is based on the covariant perturbation theory of heat kernels, with the Born approximation. The comparison shows that the scattering method based on covariant perturbation theory is a better approximation than the Born approximation.

Besides applying the heat-kernel method to scattering problems, on the other hand, by the method suggested in the present paper, we can also apply the scattering method to the heat kernel theory. In this paper, we provide a simple example for illustrating how to calculate a heat kernel from a known scattering result; more details on this subject will be given in a subsequent work. The value of developing such a method, for example, is that though it is relatively easy to obtain a high-energy expansion of heat kernels, it is difficult to obtain a low-energy heat-kernel expansion. With the help of scattering theory, we can calculate a low-energy heat-kernel expansion from a low-energy scattering theory.

The starting point of this work, as mentioned above, is a relation between the heat-kernel method and the scattering spectral method in quantum field theory. The heat-kernel method is important both in physics and mathematics. In physics, the heat-kernel method has important applications in, e.g., Euclidean field theory, gravitation theory, and statistical mechanics $[1,13,16-18]$. In mathematics, the heatkernel method is an important basis of the spectral geometry $[1,19]$. There is much research on the calculation of heat kernels. Besides exact solutions, there are many systematic studies on the asymptotic expansion of heat kernels [20], such as covariant perturbation theory [10-13]. With various heatkernel expansion techniques, one can obtain many approximate solutions of heat kernels. Scattering spectral method is an important quantum theory method which can be used to solve a variety of problems in quantum field theory, e.g., to characterize the spectrum of energy eigenstates in a potential background [2] and to solve the Casimir energy [21-25]. The method particular focuses on the property of the quantum vacuum.

In Sect. 2, we find a relation between partial-wave phase shifts and heat kernels. As a key step, we give a relation between partial-wave heat kernels and heat kernels. In Sect. 3 , based on the relation between partial-wave phase shifts and heat kernels given in Sect. 2, we establish an approach for the calculation of partial-wave phase shifts, based on an heatkernel expansion, the covariant perturbation theory. In Sect. 4, a comparison of the approach established in the present paper and the Born approximation is given; in particular, we compare these two methods through an exactly solvable potential. In Sect. 5, we give an example for calculating a heat kernel from a given phase shift. Conclusions and an outlook are given in Sect. 6. Moreover, an integral formula and two integral representations are given in Appendices A and B.

\section{Relation between partial-wave phase shift and heat kernel: calculating scattering phase shift from heat kernel}

The main result of the present paper is the following theorem which reveals a relation between partial-wave scattering phase shifts and heat kernels. This relation allows us to 
obtain a partial-wave phase shift from a known heat kernel directly. By this relation, what we can obtain is not only one method for scattering problems. It is in fact a series of methods for scattering problems: each heat kernel method leads to a method for solving scattering problems.

Theorem 1 The relation between the partial-wave scattering phase shift, $\delta_{l}(k)$, and the heat kernel, $K\left(t ; \mathbf{r}, \mathbf{r}^{\prime}\right)=$ $K\left(t ; r, \theta, \varphi, r^{\prime}, \theta^{\prime}, \varphi^{\prime}\right)$, is

$$
\begin{aligned}
& \delta_{l}(k)=2 \pi^{2} \int_{0}^{\infty} r^{2} \mathrm{~d} r \int_{-1}^{1} \mathrm{~d} \cos \gamma P_{l}(\cos \gamma) \\
& \frac{1}{2 \pi i} \int_{c-i \infty}^{c+i \infty} \mathrm{d} t \frac{e^{k^{2} t}}{t} K^{s}\left(t ; r, \theta, \varphi, r, \theta^{\prime}, \varphi^{\prime}\right)+\delta_{l}(0),
\end{aligned}
$$

where $K^{s}\left(t ; \mathbf{r}, \mathbf{r}^{\prime}\right)$ is the scattering part of a heat kernel, $P_{l}(\cos \gamma)$ is the Legendre polynomial, and $\gamma$ is the angle between $\mathbf{r}$ and $\mathbf{r}^{\prime}$ with $\cos \gamma=\cos \theta \cos \theta^{\prime}+$ $\sin \theta \sin \theta^{\prime} \cos \left(\varphi^{\prime}-\varphi\right)$.

Notice that only the radial diagonal heat kernel, $K^{s}\left(t ; r, \theta, \varphi, r, \theta^{\prime}, \varphi^{\prime}\right)$, appears in Eq. (3). The heat kernel $K\left(t ; \mathbf{r}, \mathbf{r}^{\prime}\right)$ is split into three parts: $K\left(t ; \mathbf{r}, \mathbf{r}^{\prime}\right)=$ $K^{s}\left(t ; \mathbf{r}, \mathbf{r}^{\prime}\right)+K^{b}\left(t ; \mathbf{r}, \mathbf{r}^{\prime}\right)+K^{f}\left(t ; \mathbf{r}, \mathbf{r}^{\prime}\right)$. The free part of a heat kernel $K^{f}\left(t ; \mathbf{r}, \mathbf{r}^{\prime}\right)=K^{(0)}\left(t ; \mathbf{r}, \mathbf{r}^{\prime}\right)$ is the heat kernel of the operator $D=-\nabla^{2}$; the bound part of a heat kernel corresponds to the bound state, if it exists, of the system, which, in the spectral representation, is $K^{b}\left(t ; \mathbf{r}, \mathbf{r}^{\prime}\right)=\sum_{\text {bound states }} e^{-\lambda t} \psi_{\lambda}(\mathbf{r}) \psi_{\lambda}^{*}\left(\mathbf{r}^{\prime}\right) ;$ the scattering part of a heat kernel corresponds to the scattering state of the system, which, in the spectral representation, is $K^{s}\left(t ; \mathbf{r}, \mathbf{r}^{\prime}\right)=\sum_{\text {scattering states }} e^{-\lambda t} \psi_{\lambda}(\mathbf{r}) \psi_{\lambda}^{*}\left(\mathbf{r}^{\prime}\right)$ [14]. Note that $\delta_{l}(0)=\pi / 2$ if there is a half-bound state and $\delta_{l}(0)=0$ if there is no half-bound state [14].

The remaining task of this section is to prove this theorem. In order to prove the theorem, we need to first find a relation between partial-wave heat kernels and heat kernels.

2.1 Relation between partial-wave heat kernel and heat kernel

As mentioned above, the heat kernel $K\left(t ; \mathbf{r}, \mathbf{r}^{\prime}\right)$ of an operator $D$ is determined by the heat equation (2) [1]. For a spherically symmetric operator $D$, the heat kernel $K\left(t ; \mathbf{r}, \mathbf{r}^{\prime}\right)=$ $K\left(t ; r, \theta, \varphi, r^{\prime}, \theta^{\prime}, \varphi^{\prime}\right)$ can be expressed as

$$
K\left(t ; \mathbf{r}, \mathbf{r}^{\prime}\right)=\sum_{n, l, m} e^{-\lambda_{n l} t} \psi_{n l m}(\mathbf{r}) \psi_{n l m}^{*}\left(\mathbf{r}^{\prime}\right)
$$

where $\lambda_{n l}$ and $\psi_{n l m}(\mathbf{r})=R_{n l}(r) Y_{l m}(\theta, \varphi)$ are the eigenvalue and eigenfunction of $D$, determined by the eigenequation $D \psi_{n l m}=\lambda_{n l} \psi_{n l m}$, where $R_{n l}(r)$ is the radial wave function and $Y_{l m}(\theta, \varphi)$ is the spherical harmonics. The global heat kernel is the trace of the local heat kernel $K\left(t ; \mathbf{r}, \mathbf{r}^{\prime}\right)$ :
$K(t)=\int \mathrm{d} \mathbf{r} K(t ; \mathbf{r}, \mathbf{r})=\sum_{n, l} e^{-\lambda_{n l} t}$

The local partial-wave heat kernel

$K_{l}\left(t ; r, r^{\prime}\right)=\sum_{n} e^{-\lambda_{n l} t} R_{n l}(r) R_{n l}\left(r^{\prime}\right)$

of the operator $D$ is the heat kernel of the $l$ th partial-wave radial operator [14]

$D_{l}=-\frac{1}{r^{2}} \frac{\mathrm{d}}{\mathrm{d} r}\left(r^{2} \frac{\mathrm{d}}{\mathrm{d} r}\right)+\frac{l(l+1)}{r^{2}}+V(r)$

which determines the radial equation $D_{l} R_{n l}=\lambda_{n l} R_{n l}$. The global partial-wave heat kernel is the trace of the local partialwave heat kernel $K_{l}\left(t ; r, r^{\prime}\right)$,

$K_{l}(t)=\int_{0}^{\infty} r^{2} \mathrm{~d} r K_{l}(t ; r, r)=\sum_{n} e^{-\lambda_{n l} t}$

Now we prove that the relation between $K_{l}\left(t ; r, r^{\prime}\right)$ and $K\left(t ; \mathbf{r}, \mathbf{r}^{\prime}\right)$ can be expressed as follows.

Lemma 1 The relation between the partial-wave heat kernel $K_{l}\left(t ; r, r^{\prime}\right)$ and the heat kernel $K\left(t ; \mathbf{r}, \mathbf{r}^{\prime}\right)=K(t ; r, \theta, \varphi$, $\left.r^{\prime}, \theta^{\prime}, \varphi^{\prime}\right)$ is

$$
\begin{aligned}
K_{l}\left(t ; r, r^{\prime}\right)= & 2 \pi \int_{-1}^{1} \mathrm{~d} \cos \gamma P_{l}(\cos \gamma) \\
& \times K\left(t ; r, \theta, \varphi, r^{\prime}, \theta^{\prime}, \varphi^{\prime}\right)
\end{aligned}
$$

and

$$
\begin{aligned}
K\left(t ; r, \theta, \varphi, r^{\prime}, \theta^{\prime}, \varphi^{\prime}\right)= & \frac{1}{4 \pi} \sum_{l}(2 l+1) \\
& \times P_{l}(\cos \gamma) K_{l}\left(t ; r, r^{\prime}\right) .
\end{aligned}
$$

Proof In a scattering with a spherically symmetric potential, the scattering wave function $\psi_{n l m}(r, \theta, \varphi)=R_{n l}(r)$ $Y_{l m}(\theta, \varphi)$. Then, by Eq. (4), the heat kernel can be expressed as

$$
\begin{aligned}
K\left(t ; r, \theta, \varphi, r^{\prime}, \theta^{\prime}, \varphi^{\prime}\right)= & \sum_{n, l} e^{-\lambda_{n l} t} R_{n l}(r) R_{n l}\left(r^{\prime}\right) \\
& \times \sum_{m=-l}^{l} Y_{l m}(\theta, \varphi) Y_{l m}^{*}\left(\theta^{\prime}, \varphi^{\prime}\right) .
\end{aligned}
$$

Using the relation [26]

$$
\begin{aligned}
& \sum_{m=-l}^{l} Y_{l m}(\theta, \varphi) Y_{l m}^{*}\left(\theta^{\prime}, \varphi^{\prime}\right) \\
& =\frac{2 l+1}{4 \pi} P_{l}\left(\cos \theta \cos \theta^{\prime}+\sin \theta \sin \theta^{\prime} \cos \left(\varphi^{\prime}-\varphi\right)\right),
\end{aligned}
$$


we obtain

$$
\begin{aligned}
K\left(t ; r, \theta, \varphi, r^{\prime}, \theta^{\prime}, \varphi^{\prime}\right)= & \frac{1}{4 \pi} \sum_{l}(2 l+1) P_{l}(\cos \gamma) \\
& \times \sum_{n} e^{-\lambda_{n l} t} R_{n l}(r) R_{n l}\left(r^{\prime}\right) .
\end{aligned}
$$

Then, by Eq. (6), we prove the relation (10).

Multiplying both sides of Eq. (10) by $P_{l^{\prime}}(\cos \gamma)$ and then integrating $\cos \gamma$ from -1 to 1 give

$$
\begin{aligned}
& \int_{-1}^{1} \mathrm{~d} \cos \gamma P_{l^{\prime}}(\cos \gamma) K\left(t ; r, \theta, \varphi, r^{\prime}, \theta^{\prime}, \varphi^{\prime}\right) \\
& =\frac{1}{4 \pi} \sum_{l}(2 l+1) \\
& \quad \times\left[\int_{-1}^{1} \mathrm{~d} \cos \gamma P_{l^{\prime}}(\cos \gamma) P_{l}(\cos \gamma)\right] K_{l}\left(t ; r, r^{\prime}\right) .
\end{aligned}
$$

Using the orthogonality of the Legendre polynomials [26]

$\int_{-1}^{1} \mathrm{~d} \cos \gamma P_{l^{\prime}}(\cos \gamma) P_{l}(\cos \gamma)=\frac{2}{2 l^{\prime}+1} \delta_{l l^{\prime}}$,

we obtain

$$
\begin{aligned}
& \int_{-1}^{1} \mathrm{~d} \cos \gamma P_{l^{\prime}}(\cos \gamma) K\left(t ; r, \theta, \varphi, r^{\prime}, \theta^{\prime}, \varphi^{\prime}\right) \\
& =\frac{1}{2 \pi} K_{l^{\prime}}\left(t ; r, r^{\prime}\right) .
\end{aligned}
$$

This proves the relation (9).

\subsection{Proof of Theorem 1}

Now, with Lemma 1, we can prove Theorem 1.

Proof In Ref. [14], we prove a relation between total phase shifts and global heat kernels,

$\delta(k)=\frac{1}{2 i} \int_{c-i \infty}^{c+i \infty} \frac{K^{s}(t)}{t} e^{k^{2} t} \mathrm{~d} t+\delta(0)$,

and a relation between partial-wave phase shifts and partialwave global heat kernels,

$\delta_{l}(k)=\frac{1}{2 i} \int_{c-i \infty}^{c+i \infty} \frac{K_{l}^{s}(t)}{t} e^{k^{2} t} \mathrm{~d} t+\delta_{l}(0)$.

Here the global heat kernel and the global partial-wave heat kernel are split into the scattering part, the bound part, and the free part: $K(t)=K^{s}(t)+K^{b}(t)+K^{f}(t)$ and $K_{l}(t)=$ $K_{l}^{s}(t)+K_{l}^{b}(t)+K_{l}^{f}(t)[14]$.
Starting from the global partial-wave heat kernel given by Eq. (8) and using the relation between partial-wave heat kernels and heat kernels given by Lemma 1, Eq. (9), we have

$$
\begin{aligned}
K_{l}(t)= & \int_{0}^{\infty} r^{2} \mathrm{~d} r K_{l}(t ; r, r) \\
= & 2 \pi \int_{0}^{\infty} r^{2} \mathrm{~d} r \int_{-1}^{1} \mathrm{~d} \cos \gamma P_{l}(\cos \gamma) \\
& K\left(t ; r, \theta, \varphi, r, \theta^{\prime}, \varphi^{\prime}\right) .
\end{aligned}
$$

Substituting Eq. (19) into Eq. (18) proves Theorem 1.

It should be noted here that the relations given by Ref. [14], Eqs. (17) and (18), only allow one to calculate the total phase shift $\delta(k)$ from a heat kernel $K(t)$ or to calculate the partial-wave phase shift $\delta_{l}(k)$ from a partial-wave heat kernel $K_{l}(t)$. Such results, however, are not useful in scattering problems, because the total phase shift $\delta(k)$ is not physically meaningful and the partial-wave heat kernel $K_{l}(t)$ is often difficult to obtain.

Nevertheless, the result given by Theorem 1, Eq. (3), allows one to calculate the partial-wave phase shift $\delta_{l}(k)$ from a heat kernel $K(t)$ rather than a partial-wave heat kernel $K_{l}(t)$. The heat kernel has been fully studied and there are many well-known results [1].

\section{Heat-kernel approach for phase shift: covariant perturbation theory}

In this section, based on the heat-kernel expansion given by the covariant perturbation theory [10-12], by the relation between partial-wave phase shifts and heat kernels given by Eq. (3), we establish an expansion for scattering phase shifts. The covariant perturbation theory is suitable for our purposes, since it provides a uniformly convergent expansion of heat kernels [10,27].

The covariant perturbation theory type expansion for a partial-wave scattering phase shift is $\delta_{l}(k)=\delta_{l}^{(1)}(k)+$ $\delta_{l}^{(2)}(k)+\cdots$ with

$$
\begin{aligned}
\delta_{l}^{(1)}(k)= & -\frac{\pi}{2} \int_{0}^{\infty} r \mathrm{~d} r V(r) J_{l+1 / 2}^{2}(k r), \\
\delta_{l}^{(2)}(k)= & -\frac{\pi^{2}}{2} \int_{0}^{\infty} r \mathrm{~d} r J_{l+1 / 2}(k r) Y_{l+1 / 2}(k r) V(r) \\
& \times \int_{0}^{r} r^{\prime} \mathrm{d} r^{\prime} J_{l+1 / 2}^{2}\left(k r^{\prime}\right) V\left(r^{\prime}\right),
\end{aligned}
$$

where $J_{v}(z)$ and $Y_{v}(z)$ are the Bessel functions of the first and second kinds, respectively [26].

A detailed calculation is as follows. 
3.1 Covariant perturbation theory for heat-kernel expansion

The heat-kernel expansion is systematically studied in the covariant perturbation theory [10-12]. The heat-kernel expansion given by covariant perturbation theory reads $[4,6]$

$$
\begin{aligned}
K( & \left.t ; \mathbf{r}, \mathbf{r}^{\prime}\right)=K^{(0)}\left(t ; \mathbf{r}, \mathbf{r}^{\prime}\right)+K^{(1)}\left(t ; \mathbf{r}, \mathbf{r}^{\prime}\right) \\
& +K^{(2)}\left(t ; \mathbf{r}, \mathbf{r}^{\prime}\right)+\cdots \\
= & \langle\mathbf{r}| e^{-H_{0} t}+(-t) \int_{0}^{\infty} \mathrm{d} \alpha_{1} \mathrm{~d} \alpha_{2} \delta\left(1-\alpha_{1}-\alpha_{2}\right) \\
& \times e^{-\alpha_{1} H_{0} t} V e^{-\alpha_{2} H_{0} t} \\
& +(-t)^{2} \int_{0}^{\infty} \mathrm{d} \alpha_{1} \mathrm{~d} \alpha_{2} \mathrm{~d} \alpha_{3} \delta\left(1-\alpha_{1}-\alpha_{2}-\alpha_{3}\right) e^{-\alpha_{1} H_{0} t} \\
& \times V e^{-\alpha_{2} H_{0} t} V e^{-\alpha_{3} H_{0} t}+\cdots\left|\mathbf{r}^{\prime}\right\rangle
\end{aligned}
$$

where

$K^{(0)}\left(t ; \mathbf{r}, \mathbf{r}^{\prime}\right)=\left\langle\mathbf{r}\left|e^{-H_{0} t}\right| \mathbf{r}^{\prime}\right\rangle=\frac{1}{(4 \pi t)^{3 / 2}} e^{-\left|\mathbf{r}-\mathbf{r}^{\prime}\right|^{2} /(4 t)}$

is the zero-order (free) heat kernel. Substituting the zeroorder heat kernel (23) into Eq. (22), we obtain the first two orders of a heat kernel,

$$
\begin{aligned}
& K^{(1)}\left(t ; \mathbf{r}, \mathbf{r}^{\prime}\right)=\langle\mathbf{r}|(-t) \int_{0}^{\infty} \mathrm{d} \alpha_{1} \mathrm{~d} \alpha_{2} \delta\left(1-\alpha_{1}-\alpha_{2}\right) \\
& \times e^{-\alpha_{1} H_{0} t} V e^{-\alpha_{2} H_{0} t}\left|\mathbf{r}^{\prime}\right\rangle \\
& =-\int_{0}^{t} \mathrm{~d} \tau \int \mathrm{d}^{3} y V(\mathbf{y}) \frac{\exp \left(-\frac{1}{4(t-\tau)}|\mathbf{r}-\mathbf{y}|^{2}\right)}{[4 \pi(t-\tau)]^{3 / 2}} \\
& \quad \times \frac{\exp \left(-\frac{1}{4 \tau}\left|\mathbf{y}-\mathbf{r}^{\prime}\right|^{2}\right)}{(4 \pi \tau)^{3 / 2}}
\end{aligned}
$$

and

$$
\begin{aligned}
K^{(2)} & \left(t ; \mathbf{r}, \mathbf{r}^{\prime}\right)=\langle\mathbf{r}|(-t)^{2} \\
& \times \int_{0}^{\infty} \mathrm{d} \alpha_{1} \mathrm{~d} \alpha_{2} \mathrm{~d} \alpha_{3} \delta\left(1-\alpha_{1}-\alpha_{2}-\alpha_{3}\right) \\
& \times e^{-\alpha_{1} H_{0} t} V e^{-\alpha_{2} H_{0} t} V e^{-\alpha_{3} H_{0} t}\left|\mathbf{r}^{\prime}\right\rangle \\
= & \int \mathrm{d}^{3} y V(\mathbf{y}) \int \mathrm{d}^{3} z V(\mathbf{z}) \int_{0}^{t} \mathrm{~d} \tau \int_{0}^{\tau} \mathrm{d} \tau^{\prime} \\
& \times \frac{\exp \left(-\frac{1}{4(t-\tau)}|\mathbf{r}-\mathbf{y}|^{2}\right)}{[4 \pi(t-\tau)]^{3 / 2}} \\
& \times \frac{\exp \left(-\frac{1}{4\left(\tau-\tau^{\prime}\right)}|\mathbf{y}-\mathbf{z}|^{2}\right)}{\left[4 \pi\left(\tau-\tau^{\prime}\right)\right]^{3 / 2}} \frac{\exp \left(-\frac{1}{4 \tau^{\prime}}\left|\mathbf{z}-\mathbf{r}^{\prime}\right|^{2}\right)}{\left(4 \pi \tau^{\prime}\right)^{3 / 2}} .
\end{aligned}
$$

For the spherical potentials $V(\mathbf{r})=V(r), K^{(1)}\left(t ; \mathbf{r}, \mathbf{r}^{\prime}\right)$ and $K^{(2)}\left(t ; \mathbf{r}, \mathbf{r}^{\prime}\right)$ given by Eqs. (24) and (25) become

$$
\begin{gathered}
K^{(1)}\left(t ; r, r^{\prime}, \gamma\right)=-\int_{0}^{\infty} y^{2} \mathrm{~d} y V(y) \int \mathrm{d} \Omega_{y} \int_{0}^{t} \mathrm{~d} \tau \\
\times \frac{\exp \left(-\frac{1}{4(t-\tau)}\left(r^{2}+y^{2}-2 r y \cos \gamma_{\mathbf{r y}}\right)\right)}{[4 \pi(t-\tau)]^{3 / 2}} \\
\times \frac{\exp \left(-\frac{1}{4 \tau}\left(r^{\prime 2}+y^{2}-2 r^{\prime} y \cos \gamma_{\mathbf{r}^{\prime} \mathbf{y}}\right)\right)}{(4 \pi \tau)^{3 / 2}}
\end{gathered}
$$

and

$$
\begin{aligned}
& K^{(2)}\left(t ; r, r^{\prime}, \gamma\right)=\int_{0}^{\infty} y^{2} \mathrm{~d} y V(y) \int \mathrm{d} \Omega_{y} \int_{0}^{\infty} z^{2} \mathrm{~d} z V(z) \int \mathrm{d} \Omega_{z} \\
& \times \int_{0}^{t} \mathrm{~d} \tau \int_{0}^{\tau} \mathrm{d} \tau^{\prime} \frac{\exp \left(-\frac{1}{4(t-\tau)}\left(r^{2}+y^{2}-2 r y \cos \gamma_{\mathbf{r y}}\right)\right)}{[4 \pi(t-\tau)]^{3 / 2}} \\
& \times \frac{\exp \left(-\frac{1}{4\left(\tau-\tau^{\prime}\right)}\left(y^{2}+z^{2}-2 y z \cos \gamma_{\mathbf{y z}}\right)\right)}{\left[4 \pi\left(\tau-\tau^{\prime}\right)\right]^{3 / 2}} \\
& \times \frac{\exp \left(-\frac{1}{4 \tau^{\prime}}\left(z^{2}+r^{\prime 2}-2 z r^{\prime} \cos \gamma_{\mathbf{z r}}\right)\right)}{\left(4 \pi \tau^{\prime}\right)^{3 / 2}}
\end{aligned}
$$

where $\gamma$ is the angle between $\mathbf{r}$ and $\mathbf{r}^{\prime}, \gamma_{\mathbf{r y}}$ is the angle between $\mathbf{r}$ and $\mathbf{y}, \gamma_{\mathbf{r}^{\prime} \mathbf{y}}$ is the angle between $\mathbf{r}^{\prime}$ and $\mathbf{y}, \gamma_{\mathbf{y z}}$ is the angle between $\mathbf{y}$ and $\mathbf{z}$, and $\gamma_{\mathbf{z r}}$ is the angle between $\mathbf{z}$ and $\mathbf{r}^{\prime}$.

\subsection{First-order phase shift $\delta_{l}^{(1)}(k)$}

In this section, we calculate the first-order phase shift in the frame of covariant perturbation theory.

The first-order phase shift $\delta_{l}^{(1)}(k)$ can be obtained by substituting the first-order heat kernel given by covariant perturbation theory, Eq. (26), into the relation between partialwave phase shifts and heat kernels, Eq. (3), and taking $r^{\prime}=r$ (radial diagonal):

$$
\begin{gathered}
\delta_{l}^{(1)}(k)=-2 \pi^{2} \int_{0}^{\infty} r^{2} \mathrm{~d} r \frac{1}{2 \pi i} \int_{c-i \infty}^{c+i \infty} \mathrm{d} t \frac{e^{k^{2} t}}{t} \int_{0}^{t} \mathrm{~d} \tau \\
\times \int_{0}^{\infty} y^{2} \mathrm{~d} y V(y) \frac{\exp \left(-\frac{r^{2}+y^{2}}{4(t-\tau)}\right)}{[4 \pi(t-\tau)]^{3 / 2}} \frac{\exp \left(-\frac{r^{2}+y^{2}}{4 \tau}\right)}{(4 \pi \tau)^{3 / 2}} \mathcal{I}_{1},
\end{gathered}
$$

where $\mathcal{I}_{1}$ is an integral with respect to the angle,

$$
\begin{aligned}
\mathcal{I}_{1} & =\int_{-1}^{1} \mathrm{~d} \cos \gamma P_{l}(\cos \gamma) \int \mathrm{d} \Omega_{y} \\
& \times \exp \left(\frac{r y}{2(t-\tau)} \cos \gamma_{\mathbf{r y}}\right) \exp \left(\frac{r y}{2 \tau} \cos \gamma_{\mathbf{r}^{\prime} \mathbf{y}}\right) .
\end{aligned}
$$

To calculate $\mathcal{I}_{1}$, we use the expansion [26]

$e^{i z \cos a}=\sum_{l=0}^{\infty}(2 l+1) i^{l} \sqrt{\frac{\pi}{2 z}} J_{l+1 / 2}(z) P_{l}(\cos a)$ 
to rewrite $\mathcal{I}_{1}$ as

$$
\begin{aligned}
\mathcal{I}_{1}= & \sum_{l_{1}=0}\left(2 l_{1}+1\right) i^{l_{1}} j_{l_{1}}\left(\frac{r y}{i 2(t-\tau)}\right) \\
& \times \sum_{l_{2}=0}\left(2 l_{2}+1\right) i^{l_{2}} j_{l_{2}}\left(\frac{r y}{i 2 \tau}\right) \int_{-1}^{1} \mathrm{~d} \cos \gamma P_{l}(\cos \gamma) \\
& \times \int \mathrm{d} \Omega_{y} P_{l_{1}}\left(\cos \gamma_{\mathbf{r y}}\right) P_{l_{2}}\left(\cos \gamma_{\mathbf{r}^{\prime} \mathbf{y}}\right)
\end{aligned}
$$

where $j_{v}(z)=\sqrt{\pi /(2 z)} J_{v+1 / 2}(z)$ is the spherical Bessel function of the first kind. Without loss of generality, we choose $\mathbf{r}^{\prime}=\left(r^{\prime}, 0,0\right)$ and then $\gamma_{\mathbf{r}^{\prime} \mathbf{y}}=\theta_{y}$ and $\gamma=\theta_{r}$. Now, the integral with respect to $\Omega_{y}$ can be worked out directly by using the integral formula (A.1) given in Appendix A:

$$
\begin{aligned}
& \int \mathrm{d} \Omega_{y} P_{l_{1}}\left(\cos \gamma_{\mathbf{r y}}\right) P_{l_{2}}\left(\cos \gamma_{\mathbf{r}^{\prime} \mathbf{y}}\right) \\
& =\int \mathrm{d} \Omega_{y} P_{l_{1}}\left(\cos \gamma_{\mathbf{r y}}\right) P_{l_{2}}\left(\cos \theta_{y}\right) \\
& =P_{l_{1}}\left(\cos \theta_{r}\right) \frac{4 \pi}{2 l_{1}+1} \delta_{l_{1}, l_{2}} .
\end{aligned}
$$

The integral with respect to $\gamma\left(=\theta_{r}\right)$, then, can also be worked out by using the orthogonality of the Legendre polynomials $\int_{-1}^{1} d x P_{l^{\prime}}(x) P_{l}(x)=2 /\left(2 l^{\prime}+1\right) \delta_{l l^{\prime}}[26]$ :

$$
\begin{aligned}
& \int_{-1}^{1} \mathrm{~d} \cos \theta_{r} P_{l}\left(\cos \theta_{r}\right) P_{l_{1}}\left(\cos \theta_{r}\right) \frac{4 \pi}{2 l_{1}+1} \delta_{l_{1}, l_{2}} \\
& \quad=\frac{8 \pi}{(2 l+1)^{2}} \delta_{l, l_{1}} \delta_{l_{1}, l_{2}} .
\end{aligned}
$$

By Eqs. (32) and (33), we obtain

$$
\begin{aligned}
\mathcal{I}_{1}= & \sum_{l_{1}=0}\left(2 l_{1}+1\right) i^{l_{1}} j_{l_{1}}\left(\frac{r y}{i 2(t-\tau)}\right) \\
& \times \sum_{l_{2}=0}\left(2 l_{2}+1\right) i^{l_{2}} j_{l_{2}}\left(\frac{r y}{i 2 \tau}\right) \frac{8 \pi}{(2 l+1)^{2}} \delta_{l, l_{1}} \delta_{l_{1}, l_{2}} \\
= & 8 \pi i^{2 l} j_{l}\left(\frac{r y}{i 2(t-\tau)}\right) j_{l}\left(\frac{r y}{i 2 \tau}\right) .
\end{aligned}
$$

Substituting Eq. (34) into Eq. (28) gives

$\delta_{l}^{(1)}(k)=-2 \pi^{2} \frac{1}{2 \pi i} \int_{c-i \infty}^{c+i \infty} \mathrm{d} t \frac{e^{k^{2} t}}{t} \int_{0}^{t} \mathrm{~d} \tau \int_{0}^{\infty} y^{2} \mathrm{~d} y V(y) \mathcal{I}_{2}$,

where

$$
\begin{aligned}
\mathcal{I}_{2}= & 8 \pi i^{2 l} \int_{0}^{\infty} r^{2} \mathrm{~d} r \frac{\exp \left(-\frac{r^{2}+y^{2}}{4(t-\tau)}\right)}{[4 \pi(t-\tau)]^{3 / 2}} \frac{\exp \left(-\frac{r^{2}+y^{2}}{4 \tau}\right)}{(4 \pi \tau)^{3 / 2}} \\
& \times j_{l}\left(\frac{r y}{i 2(t-\tau)}\right) j_{l}\left(\frac{r y}{i 2 \tau}\right) .
\end{aligned}
$$

To calculate the integral $\mathcal{I}_{2}$, we use the integral representation, Eq. (B.1), given in Appendix B to represent the factor $j_{l}\left(\frac{r y}{i 2(t-\tau)}\right) j_{l}\left(\frac{r y}{i 2 \tau}\right)$ as

$$
\begin{aligned}
& j_{l}\left(\frac{r y}{i 2(t-\tau)}\right) j_{l}\left(\frac{r y}{i 2 \tau}\right)=\frac{1}{2} \int_{-1}^{1} \mathrm{~d} \cos \theta \\
& \times \frac{\sin \sqrt{\left[\frac{r y}{i 2(t-\tau)}\right]^{2}+\left(\frac{r y}{i 2 \tau}\right)^{2}-2 \frac{r y}{i 2(t-\tau)} \frac{r y}{i 2 \tau} \cos \theta}}{\sqrt{\left[\frac{r y}{i 2(t-\tau)}\right]^{2}+\left(\frac{r y}{i 2 \tau}\right)^{2}-2 \frac{r y}{i 2(t-\tau)} \frac{r y}{i 2 \tau} \cos \theta}} P_{l}(\cos \theta) .
\end{aligned}
$$

Substituting the integral representation (37) into Eq. (36) and working out the integral give

$$
\begin{aligned}
\mathcal{I}_{2}= & 4 \pi i^{2 l} \int_{-1}^{1} \mathrm{~d} \cos \theta P_{l}(\cos \theta) \int_{0}^{\infty} r^{2} \mathrm{~d} r \frac{\exp \left(-\frac{r^{2}+y^{2}}{4(t-\tau)}\right)}{[4 \pi(t-\tau)]^{3 / 2}} \\
& \times \frac{\exp \left(-\frac{r^{2}+y^{2}}{4 \tau}\right)}{(4 \pi \tau)^{3 / 2}} \frac{\sin \sqrt{\left[\frac{r y}{i 2(t-\tau)}\right]^{2}+\left(\frac{r y}{i 2 \tau}\right)^{2}-2 \frac{r y}{i 2(t-\tau)} \frac{r y}{i 2 \tau} \cos \theta}}{\sqrt{\left[\frac{r y}{i 2(t-\tau)}\right]^{2}+\left(\frac{r y}{i 2 \tau}\right)^{2}-2 \frac{r y}{i 2(t-\tau)} \frac{r y}{i 2 \tau} \cos \theta}} \\
= & \frac{i^{2 l}}{(4 \pi t)^{3 / 2}} \int_{-1}^{1} \mathrm{~d} \cos \theta P_{l}(\cos \theta) \exp \left(-\frac{y^{2}}{2 t}(\cos \theta+1)\right) \cdot
\end{aligned}
$$

Substituting Eq. (38) into Eq. (35) and performing the integral with respect to $\tau$, we have

$$
\begin{aligned}
& \delta_{l}^{(1)}(k)=-\frac{\sqrt{\pi}}{4} i^{2 l} \int_{0}^{\infty} y^{2} \mathrm{~d} y V(y) \frac{1}{2 \pi i} \\
& \times \int_{c-i \infty}^{c+i \infty} \mathrm{d} t \frac{e^{k^{2} t}}{t} \frac{1}{\sqrt{t}} e^{-y^{2} /(2 t)} \\
& \times \int_{-1}^{1} \mathrm{~d} \cos \theta P_{l}(\cos \theta) \exp \left(-\frac{y^{2} \cos \theta}{2 t}\right) .
\end{aligned}
$$

Using the expansion $\exp \left(-y^{2} \cos \theta /(2 t)\right)=\sum_{l=0}(2 l+1)$ $i^{l} j_{l}\left(i \frac{y^{2}}{2 t}\right) P_{l}(\cos \theta)$ [see Eq. (30)] and the orthogonality of the Legendre polynomials, we can work out the integral:

$$
\begin{aligned}
& \int_{-1}^{1} \mathrm{~d} \cos \theta P_{l}(\cos \theta) \exp \left(-\frac{y^{2} \cos \theta}{2 t}\right) \\
& =\sum_{l^{\prime}=0}\left(2 l^{\prime}+1\right) i^{l^{\prime}} j_{l^{\prime}}\left(i \frac{y^{2}}{2 t}\right) \\
& \quad \times \int_{-1}^{1} \mathrm{~d} \cos \theta P_{l}(\cos \theta) P_{l^{\prime}}(\cos \theta) \\
& =2 i^{l} j_{l}\left(i \frac{y^{2}}{2 t}\right)=i^{2 l} \frac{2 \sqrt{\pi t}}{y} I_{l+1 / 2}\left(\frac{y^{2}}{2 t}\right),
\end{aligned}
$$

where $I_{v}(z)$ is the modified Bessel function of the first kind and the relation $j_{v}(z)=\sqrt{\pi /(2 z)} i^{l} I_{v+1 / 2}(z)$ is used. Substituting Eq. (40) into Eq. (39), we have 


$$
\begin{aligned}
\delta_{l}^{(1)}(k)= & -\frac{\pi}{2} \int_{0}^{\infty} y \mathrm{~d} y V(y) \frac{1}{2 \pi i} \int_{c-i \infty}^{c+i \infty} \mathrm{d} t \frac{e^{k^{2} t}}{t} \\
& \times e^{-y^{2} /(2 t)} I_{l+1 / 2}\left(\frac{y^{2}}{2 t}\right) .
\end{aligned}
$$

Finally, by performing the inverse Laplace transformation in Eq. (41),

$\frac{1}{2 \pi i} \int_{c-i \infty}^{c+i \infty} \mathrm{d} t \frac{e^{k^{2} t}}{t} e^{-r^{2} /(2 t)} I_{l+1 / 2}\left(\frac{r^{2}}{2 t}\right)=J_{l+1 / 2}^{2}(k r)$,

the first-order phase shift given by the covariant perturbation theory, Eq. (20), is obtained.

\subsection{Second-order phase shift $\delta_{l}^{(2)}(k)$}

In this section, we calculate the second-order phase shift in the frame of covariant perturbation theory.

The second-order phase shift $\delta_{l}^{(2)}(k)$ can be obtained by substituting the second-order heat kernel given by covariant perturbation theory, Eq. (27), into the relation between partial-wave phase shifts and heat kernels, Eq. (3), and taking $r^{\prime}=r$ :

$$
\begin{aligned}
& \delta_{l}^{(2)}(k)=2 \pi^{2} \int_{0}^{\infty} r^{2} \mathrm{~d} r \frac{1}{2 \pi i} \int_{c-i \infty}^{c+i \infty} \mathrm{d} t \frac{e^{k^{2} t}}{t} \int_{0}^{t} \mathrm{~d} \tau \int_{0}^{\tau} \mathrm{d} \tau^{\prime} \\
& \times \int_{0}^{\infty} y^{2} \mathrm{~d} y V(y) \int_{0}^{\infty} z^{2} \mathrm{~d} z V(z) \frac{\exp \left(-\frac{r^{2}+y^{2}}{4(t-\tau)}\right)}{[4 \pi(t-\tau)]^{3 / 2}} \\
& \times \frac{\exp \left(-\frac{y^{2}+z^{2}}{4\left(\tau-\tau^{\prime}\right)}\right)}{\left[4 \pi\left(\tau-\tau^{\prime}\right)\right]^{3 / 2}} \frac{\exp \left(-\frac{z^{2}+r^{2}}{4 \tau^{\prime}}\right)}{\left(4 \pi \tau^{\prime}\right)^{3 / 2}} \mathcal{I}_{3},
\end{aligned}
$$

where

$$
\begin{aligned}
\mathcal{I}_{3}= & \int_{-1}^{1} \mathrm{~d} \cos \gamma P_{l}(\cos \gamma) \int \mathrm{d} \Omega_{y} \int \mathrm{d} \Omega_{z} \exp \left(\frac{r y \cos \gamma_{\mathbf{r y}}}{2(t-\tau)}\right) \\
& \times \exp \left(\frac{y z \cos \gamma_{\mathbf{y z}}}{2\left(\tau-\tau^{\prime}\right)}\right) \exp \left(\frac{z r \cos \gamma_{\mathbf{z r}}}{2 \tau^{\prime}}\right) .
\end{aligned}
$$

Using Eq. (30), we rewrite the integral $\mathcal{I}_{3}$ as

$$
\begin{aligned}
\mathcal{I}_{3}= & \sum_{l_{1}=0}\left(2 l_{1}+1\right) i^{l_{1}} j_{l_{1}}\left(\frac{r y}{i 2(t-\tau)}\right) \\
& \times \sum_{l_{2}=0}\left(2 l_{2}+1\right) i^{l_{2}} j_{l_{2}}\left(\frac{y z}{i 2\left(\tau-\tau^{\prime}\right)}\right) \\
& \times \sum_{l_{3}=0}\left(2 l_{3}+1\right) i^{l_{3}} j_{l_{3}}\left(\frac{z r}{i 2 \tau^{\prime}}\right) \\
& \times \int_{-1}^{1} \mathrm{~d} \cos \gamma P_{l}(\cos \gamma) \int d \Omega_{y} \\
& \times \int_{\mathrm{d}} \Omega_{z} P_{l_{1}}\left(\cos \gamma_{\mathbf{r y}}\right) P_{l_{2}}\left(\cos \gamma_{\mathbf{y z}}\right) P_{l_{3}}\left(\cos \gamma_{\mathbf{z r}}\right) .
\end{aligned}
$$

Without loss of generality, we choose $\mathbf{r}^{\prime}=\left(r^{\prime}, 0,0\right)$ and then we have $\gamma_{\mathbf{z r}^{\prime}}=\theta_{z}$. The integral with respect to $\Omega_{z}$ can then be worked out by use of the integral formula, Eq. (A.1), given in Appendix A:

$$
\begin{aligned}
& \int \mathrm{d} \Omega_{z} P_{l_{2}}\left(\cos \gamma_{\mathbf{y z}}\right) P_{l_{3}}\left(\cos \gamma_{\mathbf{z} \mathbf{r}^{\prime}}\right) \\
& =\int \mathrm{d} \Omega_{z} P_{l_{2}}\left(\cos \gamma_{\mathbf{y z}}\right) P_{l_{3}}\left(\cos \theta_{z}\right) \\
& =P_{l_{2}}\left(\cos \theta_{y}\right) \frac{4 \pi}{2 l_{2}+1} \delta_{l_{2}, l_{3}} .
\end{aligned}
$$

The integral with respect to $\Omega_{y}$ also can be integrated directly by Eq. (A.1),

$$
\begin{gathered}
\int \mathrm{d} \Omega_{y} P_{l_{1}}\left(\cos \gamma_{\mathbf{r y}}\right) P_{l_{2}}\left(\cos \theta_{y}\right) \frac{4 \pi}{2 l_{2}+1} \delta_{l_{2}, l_{3}} \\
=P_{l_{1}}\left(\cos \theta_{r}\right) \frac{4 \pi}{2 l_{1}+1} \delta_{l_{1}, l_{2}} \frac{4 \pi}{2 l_{2}+1} \delta_{l_{2}, l_{3}} .
\end{gathered}
$$

Then, performing the integral with respect to $\gamma\left(\gamma=\theta_{r}\right.$ when $\left.\mathbf{r}^{\prime}=\left(r^{\prime}, 0,0\right)\right)$ in Eq. (45), we have

$$
\begin{aligned}
& \frac{4 \pi}{2 l_{1}+1} \delta_{l_{1}, l_{2}} \frac{4 \pi}{2 l_{2}+1} \delta_{l_{2}, l_{3}} \int_{-1}^{1} \mathrm{~d} \cos \theta_{r} P_{l}\left(\cos \theta_{r}\right) P_{l_{1}}\left(\cos \theta_{r}\right) \\
& =\frac{32 \pi^{2}}{(2 l+1)^{3}} \delta_{l, l_{1}} \delta_{l_{1}, l_{2}} \delta_{l_{2}, l_{3}}
\end{aligned}
$$

By Eqs. (46), (47), and (48), we have

$$
\mathcal{I}_{3}=32 \pi^{2} i^{3 l} j_{l}\left(\frac{r y}{i 2(t-\tau)}\right) j_{l}\left(\frac{y z}{i 2\left(\tau-\tau^{\prime}\right)}\right) j_{l}\left(\frac{z r}{i 2 \tau^{\prime}}\right) .
$$

Substituting Eq. (49) into Eq. (43) gives

$$
\begin{aligned}
& \delta_{l}^{(2)}(k)=64 \pi^{4} i^{3 l} \frac{1}{2 \pi i} \int_{c-i \infty}^{c+i \infty} \mathrm{d} t \frac{e^{k^{2} t}}{t} \int_{0}^{t} \mathrm{~d} \tau \int_{0}^{\tau} \mathrm{d} \tau^{\prime} \\
& \times \int_{0}^{\infty} y^{2} \mathrm{~d} y V(y) \int_{0}^{\infty} z^{2} \mathrm{~d} z V(z) \\
& \times \frac{\exp \left(-\frac{y^{2}+z^{2}}{4\left(\tau-\tau^{\prime}\right)}\right)}{\left[4 \pi\left(\tau-\tau^{\prime}\right)\right]^{3 / 2}} j_{l}\left(\frac{y z}{i 2\left(\tau-\tau^{\prime}\right)}\right) \\
& \times \int_{0}^{\infty} r^{2} \mathrm{~d} r \frac{\exp \left(-\frac{r^{2}+y^{2}}{4(t-\tau)}\right)}{[4 \pi(t-\tau)]^{3 / 2}} \frac{\exp \left(-\frac{z^{2}+r^{2}}{4 \tau^{\prime}}\right)}{\left(4 \pi \tau^{\prime}\right)^{3 / 2}} \\
& \times j_{l}\left(\frac{r y}{i 2(t-\tau)}\right) j_{l}\left(\frac{z r}{i 2 \tau^{\prime}}\right) .
\end{aligned}
$$

To perform the integral with respect to $r$, by using the integral representation (B.1) given in Appendix B, we rewrite 


$$
\begin{aligned}
& j_{l}\left(\frac{r y}{i 2(t-\tau)}\right) j_{l}\left(\frac{z r}{i 2 \tau^{\prime}}\right)=\frac{1}{2} \int_{-1}^{1} \mathrm{~d} \cos \theta \\
& \times \frac{\sin \sqrt{\left[\frac{r y}{i 2(t-\tau)}\right]^{2}+\left(\frac{z r}{i 2 \tau^{\prime}}\right)^{2}-2 \frac{r y}{i 2(t-\tau)} \frac{z r}{i 2 \tau^{\prime}} \cos \theta}}{\sqrt{\left[\frac{r y}{i 2(t-\tau)}\right]^{2}+\left(\frac{z r}{i 2 \tau^{\prime}}\right)^{2}-2 \frac{r y}{i 2(t-\tau)} \frac{z r}{i 2 \tau^{\prime}} \cos \theta}} P_{l}(\cos \theta) .
\end{aligned}
$$

Then the integral with respect to $r$ can be worked out,

$$
\begin{aligned}
& \int_{0}^{\infty} r^{2} \mathrm{~d} r \frac{\exp \left(-\frac{r^{2}+y^{2}}{4(t-\tau)}\right)}{[4 \pi(t-\tau)]^{3 / 2}} \frac{\exp \left(-\frac{z^{2}+r^{2}}{4 \tau^{\prime}}\right)}{\left(4 \pi \tau^{\prime}\right)^{3 / 2}} \\
& \times j_{l}\left(\frac{r y}{i 2(t-\tau)}\right) j_{l}\left(\frac{z r}{i 2 \tau^{\prime}}\right) \\
& =\frac{1}{2} \int_{-1}^{1} \mathrm{~d} \cos \theta P_{l}(\cos \theta) \\
& \times \int_{0}^{\infty} r^{2} \mathrm{~d} r \frac{\exp \left(-\frac{r^{2}+y^{2}}{4(t-\tau)}\right)}{[4 \pi(t-\tau)]^{3 / 2}} \frac{\exp \left(-\frac{z^{2}+r^{2}}{4 \tau^{\prime}}\right)}{\left(4 \pi \tau^{\prime}\right)^{3 / 2}} \\
& \times \frac{\sin \sqrt{\left[\frac{r y}{i 2(t-\tau)}\right]^{2}+\left(\frac{z r}{i 2 \tau^{\prime}}\right)^{2}-2 \frac{r y}{i 2(t-\tau)} \frac{z r}{i 2 \tau^{\prime}} \cos \theta}}{\sqrt{\left[\frac{r y}{i 2(t-\tau)}\right]^{2}+\left(\frac{z r}{i 2 \tau^{\prime}}\right)^{2}-2 \frac{r y}{i 2(t-\tau)} \frac{z r}{i 2 \tau^{\prime}} \cos \theta}} \\
& =\frac{1}{8 \pi} \int_{-1}^{1} \mathrm{~d} \cos \theta P_{l}(\cos \theta) \frac{1}{\left[4 \pi\left(t-\tau+\tau^{\prime}\right)\right]^{3 / 2}} \\
& \times \exp \left(-\frac{y^{2}+z^{2}+2 y z \cos \theta}{4\left(t-\tau+\tau^{\prime}\right)}\right) .
\end{aligned}
$$

Substituting Eq. (52) into Eq. (50), we have

$$
\begin{aligned}
& \delta_{l}^{(2)}(k)=8 \pi^{3} i^{3 l} \frac{1}{2 \pi i} \int_{c-i \infty}^{c+i \infty} \mathrm{d} t \frac{e^{k^{2} t}}{t} \int_{0}^{t} \mathrm{~d} \tau \int_{0}^{\tau} \mathrm{d} \tau^{\prime} \\
& \times \int_{0}^{\infty} y^{2} \mathrm{~d} y V(y) \int_{0}^{\infty} z^{2} \mathrm{~d} z V(z) \frac{\exp \left(-\frac{y^{2}+z^{2}}{4\left(\tau-\tau^{\prime}\right)}\right)}{\left[4 \pi\left(\tau-\tau^{\prime}\right)\right]^{3 / 2}} \\
& \quad \times j_{l}\left(\frac{y z}{i 2\left(\tau-\tau^{\prime}\right)}\right) \frac{\exp \left(-\frac{y^{2}+z^{2}}{4\left(t-\tau+\tau^{\prime}\right)}\right)}{\left[4 \pi\left(t-\tau+\tau^{\prime}\right)\right]^{3 / 2}} \\
& \quad \times \int_{-1}^{1} \mathrm{~d} \cos \theta P_{l}(\cos \theta) \exp \left(-\frac{y z \cos \theta}{2\left(t-\tau+\tau^{\prime}\right)}\right) .
\end{aligned}
$$

Using the expansion (30) and the orthogonality of the Legendre polynomials, we have

$$
\begin{aligned}
& \int_{-1}^{1} \mathrm{~d} \cos \theta P_{l}(\cos \theta) \exp \left(-\frac{y z \cos \theta}{2\left(t-\tau+\tau^{\prime}\right)}\right) \\
& =\sum_{l^{\prime}=0}\left(2 l^{\prime}+1\right) i^{l^{\prime}} j_{l^{\prime}}\left(-\frac{y z}{i 2\left(t-\tau+\tau^{\prime}\right)}\right) \\
& \quad \times \int_{-1}^{1} \mathrm{~d} \cos \theta P_{l}(\cos \theta) P_{l^{\prime}}(\cos \theta) \\
& =2 i^{l} j_{l}\left(-\frac{y z}{i 2\left(t-\tau+\tau^{\prime}\right)}\right) .
\end{aligned}
$$

Substituting Eq. (54) into Eq. (53) and setting $T=\tau-\tau^{\prime}$, we have

$$
\begin{gathered}
\delta_{l}^{(2)}(k)=16 \pi^{3} \frac{1}{2 \pi i} \int_{c-i \infty}^{c+i \infty} \mathrm{d} t \frac{e^{k^{2} t}}{t} \int_{0}^{\infty} y^{2} \mathrm{~d} y V(y) \\
\times \int_{0}^{\infty} z^{2} \mathrm{~d} z V(z) \int_{0}^{t} \mathrm{~d} \tau \int_{0}^{\tau} \mathrm{d} T \frac{\exp \left(-\frac{y^{2}+z^{2}}{4 T}\right)}{(4 \pi T)^{3 / 2}} \\
\times \frac{\exp \left(-\frac{y^{2}+z^{2}}{4(t-T)}\right)}{[4 \pi(t-T)]^{3 / 2}} j_{l}\left(\frac{y z}{i 2 T}\right) j_{l}\left(-\frac{y z}{i 2(t-T)}\right) .
\end{gathered}
$$

Exchanging the order of integrals $\int_{0}^{t} \mathrm{~d} \tau \int_{0}^{\tau} \mathrm{d} T \rightarrow \int_{0}^{t} \mathrm{~d} T \int_{T}^{t} \mathrm{~d} \tau$ and resetting $T=\tau^{\prime}$ give

$$
\begin{gathered}
\delta_{l}^{(2)}(k)=16 \pi^{3} \frac{1}{2 \pi i} \int_{c-i \infty}^{c+i \infty} \mathrm{d} t \frac{e^{k^{2} t}}{t} \int_{0}^{\infty} y^{2} \mathrm{~d} y V(y) \\
\times \int_{0}^{\infty} z^{2} \mathrm{~d} z V(z) \int_{0}^{t} \mathrm{~d} \tau^{\prime} \int_{\tau^{\prime}}^{t} \mathrm{~d} \tau \frac{\exp \left(-\frac{y^{2}+z^{2}}{4 \tau^{\prime}}\right)}{\left(4 \pi \tau^{\prime}\right)^{3 / 2}} \\
\times \frac{\exp \left(-\frac{y^{2}+z^{2}}{4\left(t-\tau^{\prime}\right)}\right)}{\left[4 \pi\left(t-\tau^{\prime}\right)\right]^{3 / 2}} j_{l}\left(\frac{y z}{i 2 \tau^{\prime}}\right) j_{l}\left(-\frac{y z}{i 2\left(t-\tau^{\prime}\right)}\right) .
\end{gathered}
$$

Integrating with respect to $\tau$, we have

$$
\begin{aligned}
\delta_{l}^{(2)}(k)= & \frac{1}{4} \int_{0}^{\infty} y^{2} \mathrm{~d} y V(y) \int_{0}^{\infty} z^{2} \mathrm{~d} z V(z) \\
& \times \frac{1}{2 \pi i} \int_{c-i \infty}^{c+i \infty} \mathrm{d} t e^{k^{2} t} \mathcal{I}_{4},
\end{aligned}
$$

where

$$
\begin{aligned}
\mathcal{I}_{4}= & \frac{1}{t} \int_{0}^{t} \mathrm{~d} \tau^{\prime} \frac{\exp \left(-\frac{y^{2}+z^{2}}{4\left(t-\tau^{\prime}\right)}\right)}{\left(t-\tau^{\prime}\right)^{1 / 2}} \\
& \times \frac{\exp \left(-\frac{y^{2}+z^{2}}{4 \tau^{\prime}}\right)}{\tau^{\prime 3 / 2}} j_{l}\left(-\frac{y z}{i 2\left(t-\tau^{\prime}\right)}\right) j_{l}\left(\frac{y z}{i 2 \tau^{\prime}}\right) \\
= & -8 \int_{0}^{\infty} k \mathrm{~d} k e^{-k^{2} t}\left\{\begin{array}{ll}
k^{2} j_{l}(k y) n_{l}(k y) j_{l}^{2}(k z), & y>z \\
k^{2} j_{l}^{2}(k y) j_{l}(k z) n_{l}(k z), & y<z
\end{array},\right.
\end{aligned}
$$

where $n_{l}(z)$ is the spherical Bessel function of the second kind. Thus, the inverse Laplace transformation of $\mathcal{I}_{4}$ can be worked out:

$$
\begin{aligned}
& \frac{1}{2 \pi i} \int_{c-i \infty}^{c+i \infty} \mathrm{d} t e^{k^{2} t} \mathcal{I}_{4} \\
& \quad=-4\left\{\begin{array}{ll}
k^{2} j_{l}(k y) n_{l}(k y) j_{l}^{2}(k z) & y>z \\
k^{2} j_{l}^{2}(k y) j_{l}(k z) n_{l}(k z), & y<z
\end{array} .\right.
\end{aligned}
$$

Substituting Eq. (59) into Eq. (57), we have

$$
\begin{aligned}
\delta_{l}^{(2)}(k)= & -k^{2} \int_{0}^{\infty} y^{2} \mathrm{~d} y j_{l}(k y) n_{l}(k y) V(y) \\
& \times \int_{0}^{y} z^{2} \mathrm{~d} z j_{l}^{2}(k z) V(z)
\end{aligned}
$$




$$
\begin{aligned}
& -k^{2} \int_{0}^{\infty} y^{2} \mathrm{~d} y j_{l}^{2}(k y) V(y) \\
& \times \int_{y}^{\infty} z^{2} \mathrm{~d} z j_{l}(k z) n_{l}(k z) V(z) .
\end{aligned}
$$

By exchanging the order of integrals, $\int_{0}^{\infty} \mathrm{d} y \int_{y}^{\infty} \mathrm{d} z \rightarrow$ $\int_{0}^{\infty} \mathrm{d} z \int_{0}^{z} \mathrm{~d} y$, we rewrite Eq. (60) as

$$
\begin{aligned}
\delta_{l}^{(2)}(k)= & -k^{2} \int_{0}^{\infty} y^{2} \mathrm{~d} y j_{l}(k y) n_{l}(k y) V(y) \\
& \times \int_{0}^{y} z^{2} \mathrm{~d} z j_{l}^{2}(k z) V(z) \\
& -k^{2} \int_{0}^{\infty} z^{2} \mathrm{~d} z j_{l}(k z) n_{l}(k z) V(z) \\
& \times \int_{0}^{z} y^{2} \mathrm{~d} y j_{l}^{2}(k y) V(y) .
\end{aligned}
$$

Obviously, the two parts in Eq. (61) are equal. Using $j_{l}(z)=$ $\sqrt{\pi /(2 z)} J_{l+1 / 2}(z)$ and $n_{l}(z)=\sqrt{\pi /(2 z)} Y_{l+1 / 2}(z)$ gives Eq. (21).

\section{Comparison with Born approximation}

The approach for scattering problems established in the present paper is to convert a method of calculating heat kernels into a method of solving scattering problems. As an application, in Sect. 3, we suggest a method for the scattering phase shift, based on the covariant perturbation theory of heat kernels.

In scattering theory, there are many approximation methods, such as the Born approximation, the WKB method, the eikonal approximation, and the variational method [28].

In this section, we compare our method with the Born approximation.

\subsection{Comparison of first-order contribution}

For clarity, we list the result given by the above section in the following.

The first-order phase shift given by the covariant perturbation theory given in Sect. 3 reads

$\delta_{l}^{(1)}(k)_{\mathrm{cpt}}=-\frac{\pi}{2} \int_{0}^{\infty} r \mathrm{~d} r J_{l+1 / 2}^{2}(k r) V(r)$.

For comparison: the first-order phase shift given by the Born approximation reads [28]

$$
\begin{aligned}
\delta_{l}^{(1)}(k)_{\text {Born }} & =\arctan \left[-\frac{\pi}{2} \int_{0}^{\infty} r \mathrm{~d} r V(r) J_{l+1 / 2}^{2}(k r)\right] \\
& \simeq-\frac{\pi}{2} \int_{0}^{\infty} r \mathrm{~d} r J_{l+1 / 2}^{2}(k r) V(r)+\cdots .
\end{aligned}
$$

Obviously, the leading contributions of these two methods are the same (in the Born approximation, the first-order contribution is in fact $\arctan \left[-(\pi / 2) \int_{0}^{\infty} r \mathrm{~d} r V(r) J_{l+1 / 2}^{2}(k r)\right]$, but the higher contribution can be safely ignored in the firstorder contribution).

\subsection{Comparison of second-order contribution}

The second-order phase shift given by the covariant perturbation theory given in Sect. 3 [Eqs. (21) and (61)] reads

$$
\begin{aligned}
\delta_{l}^{(2)}(k)_{\mathrm{cpt}}= & -\frac{\pi^{2}}{2} \int_{0}^{\infty} r \mathrm{~d} r J_{l+1 / 2}(k r) Y_{l+1 / 2}(k r) V(r) \\
& \times \int_{0}^{r} r^{\prime} \mathrm{d} r^{\prime} J_{l+1 / 2}^{2}\left(k r^{\prime}\right) V\left(r^{\prime}\right) \\
= & -\frac{\pi^{2}}{4} \int_{0}^{\infty} r \mathrm{~d} r J_{l+1 / 2}(k r) Y_{l+1 / 2}(k r) V(r) \\
& \times \int_{0}^{r} r^{\prime} \mathrm{d} r^{\prime} J_{l+1 / 2}^{2}\left(k r^{\prime}\right) V\left(r^{\prime}\right) \\
& -\frac{\pi^{2}}{4} \int_{0}^{\infty} r \mathrm{~d} r J_{l+1 / 2}^{2}(k r) V(r) \\
& \times \int_{r}^{\infty} r^{\prime} \mathrm{d} r^{\prime} J_{l+1 / 2}\left(k r^{\prime}\right) Y_{l+1 / 2}\left(k r^{\prime}\right) V\left(r^{\prime}\right) .
\end{aligned}
$$

The second-order phase shift given by the Born approximation [28] reads

$$
\begin{aligned}
\delta_{l}^{(2)}(k)_{\text {Born }}= & \arctan \left[-\frac{\pi^{2}}{4} \int_{0}^{\infty} r \mathrm{~d} r J_{l+1 / 2}(k r) Y_{l+1 / 2}(k r) V(r)\right. \\
& \times \int_{0}^{r} r^{\prime} \mathrm{d} r^{\prime} J_{l+1 / 2}^{2}\left(k r^{\prime}\right) V\left(r^{\prime}\right) \\
& -\frac{\pi^{2}}{4} \int_{0}^{\infty} r \mathrm{~d} r J_{l+1 / 2}^{2}(k r) V(r) \\
& \left.\times \int_{r}^{\infty} r^{\prime} \mathrm{d} r^{\prime} J_{l+1 / 2}\left(k r^{\prime}\right) Y_{l+1 / 2}\left(k r^{\prime}\right) V\left(r^{\prime}\right)\right] \\
\simeq & -\frac{\pi^{2}}{4} \int_{0}^{\infty} r \mathrm{~d} r J_{l+1 / 2}(k r) Y_{l+1 / 2}(k r) V(r) \\
& \times \int_{0}^{r} r^{\prime} \mathrm{d} r^{\prime} J_{l+1 / 2}^{2}\left(k r^{\prime}\right) V\left(r^{\prime}\right) \\
& -\frac{\pi^{2}}{4} \int_{0}^{\infty} r \mathrm{~d} r J_{l+1 / 2}^{2}(k r) V(r) \\
& \times \int_{r}^{\infty} r^{\prime} \mathrm{d} r^{\prime} J_{l+1 / 2}\left(k r^{\prime}\right) Y_{l+1 / 2}\left(k r^{\prime}\right) V\left(r^{\prime}\right)+\cdots
\end{aligned}
$$

It can be directly seen that the leading contribution of the second-order Born approximation and the leading contribution of the second-order covariant perturbation theory are the same. 
4.3 Comparison through an exactly solvable potential $V(r)=\alpha / r^{2}$

In this section, we compare the two methods, the covariant perturbation theory method, and the Born approximation, through an exactly solvable potential:

$V(r)=\frac{\alpha}{r^{2}}$

Using these two approximation methods to calculate an exactly solvable potential can help us to compare them intuitively.

The phase shift for the potential (66) can be solved exactly,

$\delta_{l}=-\frac{\pi}{2}\left[\sqrt{\left(l+\frac{1}{2}\right)^{2}+\alpha}-\left(l+\frac{1}{2}\right)\right]$.

In order to compare the methods term by term, we expand the exact result (67) as $\delta_{l}=\delta_{l}^{(1)}+\delta_{l}^{(2)}+\cdots$, where

$\delta_{l}^{(1)}=-\frac{\pi \alpha}{2(2 l+1)}$,

$\delta_{l}^{(2)}=\frac{\pi \alpha^{2}}{2(2 l+1)^{3}}$.

First order The first-order contribution given by the covariant perturbation theory and the Born approximation can be directly obtained by substituting the potential (66) into Eqs. (20) and (63), respectively:

$$
\begin{aligned}
\delta_{l}^{(1)}(k)_{\mathrm{cpt}} & =-\frac{\pi \alpha}{2(2 l+1)}, \\
\delta_{l}^{(1)}(k)_{\mathrm{Born}} & =\arctan \left[-\frac{\pi \alpha}{2(2 l+1)}\right] \\
& \simeq-\frac{\pi \alpha}{2(2 l+1)}-\frac{1}{3}\left[-\frac{\pi \alpha}{2(2 l+1)}\right]^{3} .
\end{aligned}
$$

Comparing with the direct expansion of the exact solution, Eqs. (68) and (69), we can see that both results are good approximations, and the result given by covariant perturbation theory is better than the result given by the Born approximation.

Second order The second-order contribution given by the covariant perturbation theory and the Born approximation can be directly obtained by substituting the potential (66) into Eqs. (21) and (65), respectively:

$$
\begin{aligned}
\delta_{l}^{(2)}(k)_{\mathrm{cpt}} & =\frac{\pi \alpha^{2}}{2(2 l+1)^{3}}, \\
\delta_{l}^{(2)}(k)_{\mathrm{Born}} & =\arctan \left[\frac{\pi \alpha^{2}}{2(2 l+1)^{3}}\right] \\
& \simeq \frac{\pi \alpha^{2}}{2(2 l+1)^{3}}-\frac{1}{3}\left[\frac{\pi \alpha^{2}}{2(2 l+1)^{3}}\right]^{3} .
\end{aligned}
$$

Comparing with the second-order contribution, Eq. (69), we can see that, like that in the case of first-order contributions, the result given by the covariant perturbation theory is better.

\section{Calculating global heat kernel from phase shift}

The key result of this paper is a relation between partialwave phase shifts and heat kernels. Besides solving a scattering problem from a known heat kernel, obviously, we can also calculate a heat kernel from a known phase shift. Here, we only give a simple example with the potential $\alpha / r^{2}$. A systematic discussion of how to calculate heat kernels and other spectral functions, such as one-loop effective actions, vacuum energies, and spectral counting functions, from a solved scattering problem will be given elsewhere.

For the potential

$V(r)=\frac{\alpha}{r^{2}}$

the exact partial-wave phase shift is given by Eq. (67),

$\delta_{l}=-\frac{\pi}{2}\left[\sqrt{\left(l+\frac{1}{2}\right)^{2}+\alpha}-\left(l+\frac{1}{2}\right)\right]$.

By the relation between a global heat kernel and a scattering phase shift given by Ref. [14],

$K_{l}^{S}(t)=\frac{2}{\pi} t \int_{0}^{\infty} k \mathrm{~d} k \delta_{l}(k) e^{-k^{2} t}-\frac{\delta_{l}(0)}{\pi}$,

we can calculate the scattering part of the global heat kernel immediately,

$K_{l}^{s}(t)=-\frac{1}{2}\left[\sqrt{\alpha+\left(l+\frac{1}{2}\right)^{2}}-\left(l+\frac{1}{2}\right)\right]$.

In this case, the bound part of heat kernel $K_{l}^{b}(t)=0$ and the free part of heat kernel $K_{l}^{f}(t)=R / \sqrt{4 \pi t}-\frac{1}{2}\left(l+\frac{1}{2}\right)$, where $R$ is the radius of the system. The global partial-wave heat kernel then reads

$$
\begin{aligned}
K_{l}(t) & =K_{l}^{s}(t)+K_{l}^{b}(t)+K_{l}^{f}(t) \\
& =\frac{R}{\sqrt{4 \pi t}}-\frac{1}{2} \sqrt{\alpha+\left(l+\frac{1}{2}\right)^{2}} .
\end{aligned}
$$

As a comparison, we calculate the partial-wave heat kernel for $V(r)=\alpha / r^{2}$ by another approach. 
The partial-wave heat kernel of a free particle, $K_{l}^{f}$, which is the heat kernel of the radial operator $D^{\text {free }}=$ $-\frac{1}{r^{2}} \frac{\mathrm{d}}{\mathrm{d} r}\left(r^{2} \frac{\mathrm{d}}{\mathrm{d} r}\right)+\frac{l(l+1)}{r^{2}}$, can be calculated directly:

$K_{l}^{f}\left(t ; r, r^{\prime}\right)=\frac{1}{2 t \sqrt{r r^{\prime}}} \exp \left(-\frac{r^{2}+r^{\prime 2}}{4 t}\right) I_{l+1 / 2}\left(\frac{r r^{\prime}}{2 t}\right)$.

By setting $\frac{s(s+1)}{r^{2}}=\frac{l(l+1)}{r^{2}}+\frac{\alpha}{r^{2}}$, where $s=\sqrt{\alpha+(l+1 / 2)^{2}}-$ $1 / 2$, we can obtain the partial-wave heat kernel of the operator $D_{l}=-\frac{1}{r^{2}} \frac{\mathrm{d}}{\mathrm{d} r}\left(r^{2} \frac{\mathrm{d}}{\mathrm{d} r}\right)+\frac{l(l+1)}{r^{2}}+\frac{\alpha}{r^{2}}$,

$$
\begin{aligned}
K_{l}\left(t ; r, r^{\prime}\right)= & \frac{1}{2 t \sqrt{r r^{\prime}}} \exp \left(-\frac{r^{2}+r^{\prime 2}}{4 t}\right) \\
& \times I \sqrt{\alpha+(l+1 / 2)^{2}}\left(\frac{r r^{\prime}}{2 t}\right) .
\end{aligned}
$$

Taking the trace of $K_{l}\left(t ; r, r^{\prime}\right)$ gives the global partial-wave heat kernel $K_{l}(t)$ :

$$
\begin{aligned}
K_{l}(t)= & \int_{0}^{R} r^{2} \mathrm{~d} r K_{l}(t ; r, r) \\
= & \frac{R^{2(1+\eta)}}{(4 t)^{1+\eta} \Gamma(2+\eta)} \\
& \times{ }_{2} F_{2}\left(\eta+\frac{1}{2}, \eta+1 ; \eta+2,2 \eta+1 ;-\frac{R^{2}}{t}\right),
\end{aligned}
$$

where $\eta=\sqrt{(l+1 / 2)^{2}+\alpha},{ }_{p} F_{q}\left(a_{1}, a_{2} \cdots a_{p} ; b_{1}, b_{2} \cdots\right.$ $\left.b_{q} ; z\right)$ is the generalized hypergeometric function [26]. Expanding $K_{l}(t)$ at $R \rightarrow \infty$ gives

$K_{l}(t)=\frac{R}{\sqrt{4 \pi t}}-\frac{1}{2} \eta+\sqrt{t} \frac{2 \eta^{2}-\frac{1}{2}+i e^{-R^{2} / t-i \pi \eta}}{4 \sqrt{\pi} R}+\cdots$

When $R \rightarrow \infty$, one recovers the heat kernel given by Eq. (78).

\section{Conclusions and outlook}

In this paper, based on two quantum field theory methods, the heat-kernel method [1] and the scattering spectral method [2], we suggest an approach for calculating the scattering phase shift. The method suggested in the present paper is indeed a series of different methods of calculating scattering phase shifts constructed from various heat-kernel methods.

The key step is to find a relation between partial-wave phase shifts and heat kernels. This relation allows us to express a partial-wave phase shift by a heat kernel. Then each method of the calculation of heat kernels can be converted to a method of the calculation of phase shifts.

As an application, we provide a method for the calculation of phase shifts based on the covariant perturbation theory of heat kernels.

Furthermore, as emphasized above, by this approach, we can construct various methods for scattering problems with the help of various heat-kernel methods. In subsequent works, we shall construct various scattering methods by using various heat-kernel expansions.

In this paper, as a byproduct, we also provide an offdiagonal heat-kernel expansion based on the technique developed in the covariant perturbation theory for diagonal heat kernels, since the heat-kernel method for scatterings established in the present paper is based on the off-diagonal heat kernel rather than the diagonal heat kernel. It should be emphasized that many methods for calculating diagonal heat kernels can be directly applied to the calculation of offdiagonal heat kernels. That is to say, the method for calculating the diagonal heat kernel often can also be converted to a method for calculating off-diagonal heat kernels and scattering phase shifts, as we have done in the present paper. Therefore, we can construct scattering methods from many methods of diagonal heat kernels, e.g., [1,9,29].

The heat kernel theory is well studied in both mathematics and physics. Here, as examples, we list some methods on the calculation of heat kernel. In Refs. [30-32], the authors calculate the heat-kernel coefficient with different boundary conditions. In Ref. [33], using the background field method, the author calculates the fourth and fifth heat-kernel coefficients. In Refs. [34-36], the authors calculate the third coefficient by the covariant technique. In Refs. [9,37], by a string-inspired worldline path-integral method, the authors calculate the first seven heat-kernel coefficients. In Ref. [29], a direct, nonrecursive method for the calculation of heat kernels is presented. In Ref. [38], the first five heat-kernel coefficients for a general Laplace-type operator on a compact Riemannian space without boundary by the index-free notation are given. In Refs. [10-13,27,39-43], a covariant perturbation theory which yields a uniformly convergent expansion of heat kernels is established. In Refs. [44-46], a covariant pseudo-differential-operator method for calculating heat-kernel expansions in an arbitrary space dimension is given.

An important application of the method given by this paper is to solve various spectral functions by a scattering method. The problem of spectral functions is an important issue in quantum field theory $[15,47,48]$. A subsequent work on this subject is a systematic discussion of calculating heat kernels, effective actions, vacuum energies, etc., from a known phase shift. We will show that, based on scattering methods, we can obtain some new heat-kernel expansions. It is well known that though there are many discussions on the high energy heat- 
kernel expansion, the low-energy expansion of heat kernels is relatively difficult to obtain. While there are some successful low-energy scattering theories, by using the relation given in this paper we can directly obtain some low-energy results for heat kernels.

Starting from the result given by the present paper, we can study many problems. The method presented in this paper can be applied to low-dimensional scatterings. Oneand two-dimensional scatterings and their applications have been thoroughly studied, such as the transport property of low-dimensional materials [49-51]. We will also consider a systematic application of our method to relativistic scattering. The relativistic scattering is an important problem, e.g., the collision of solitons in relativistic scalar field theories [52] and the Dirac scattering in the problem of the electron properties of graphene $[53,54]$. We can also apply the method to low-temperature physics. There are many scattering problems in low-temperature physics, such as the scattering in the problem of the transition temperature of a BEC $[55,56]$ and the transport property of spin-polarized fermions at low temperature $[57,58]$.

The application of the method to inverse scattering problems is an important subject of our subsequent work. The inverse scattering problem has extreme significance in physics $[59,60]$. In practice, for example, the inverse scattering method can be applied to the problem of the BEC [61] and the Aharonov-Bohm effect [62].

In Ref. [47], we provide a method for solving for the spectral function, such as one-loop effective actions, vacuum energies, and spectral counting functions in quantum field theory. The key idea is to construct the equations obeyed by these quantities. We show that, for example, the equation of the one-loop effective action is a partial integro-differential equation. By the relation between partial-wave phase shifts and heat kernel, we can also construct an equation obeyed by phase shifts.

Moreover, in conventional scattering theory, approximate large-distance asymptotics is used to seek an explicit result. In Ref. [63], we show that such an approximate treatment is not necessary: without the large-distance asymptotics, one can still rigorously obtain an explicit result. The result presented in this paper can be directly applied to the scattering theory without large-distance asymptotics.

Acknowledgments We are very indebted to Dr G. Zeitrauman for his encouragement. This work is supported in part by NSF of China under Grant No. 11075115.

Open Access This article is distributed under the terms of the Creative Commons Attribution 4.0 International License (http://creativecomm ons.org/licenses/by/4.0/), which permits unrestricted use, distribution, and reproduction in any medium, provided you give appropriate credit to the original author(s) and the source, provide a link to the Creative Commons license, and indicate if changes were made.

Funded by SCOAP . $^{3}$

\section{Appendix A: $\int \mathrm{d} \Omega^{\prime} P_{l}(\cos \gamma) P_{l^{\prime}}\left(\cos \theta^{\prime}\right)$}

In this appendix, we provide an integral formula:

$\int \mathrm{d} \Omega^{\prime} P_{l}(\cos \gamma) P_{l^{\prime}}\left(\cos \theta^{\prime}\right)=\frac{4 \pi}{2 l+1} P_{l}(\cos \theta) \delta_{l l^{\prime}}$,

where $\gamma$ is the angle between $\mathbf{r}=(r, \theta, \phi)$ and $\mathbf{r}^{\prime}=$ $\left(r^{\prime}, \theta^{\prime}, \phi^{\prime}\right)$ and $\mathrm{d} \Omega^{\prime}=\sin \theta^{\prime} \mathrm{d} \theta^{\prime} \mathrm{d} \phi^{\prime}$.

Proof Using the integral formula [64]

$\int \mathrm{d} \Omega^{\prime} Y_{l 0}(\gamma) Y_{l^{\prime} 0}\left(\theta^{\prime}\right)=\sqrt{\frac{4 \pi}{2 l+1}} Y_{l 0}(\theta) \delta_{l l^{\prime}}$

and the relation $Y_{l 0}(\theta, \phi)=\sqrt{(2 l+1) /(4 \pi)} P_{l}(\cos \theta)$, we have

$$
\begin{aligned}
\int \mathrm{d} \Omega^{\prime} Y_{l 0}(\gamma) Y_{l^{\prime} 0}\left(\theta^{\prime}\right)= & \sqrt{\frac{2 l+1}{4 \pi}} \sqrt{\frac{2 l^{\prime}+1}{4 \pi}} \\
& \times \int \mathrm{d} \Omega^{\prime} P_{l}(\cos \gamma) P_{l^{\prime}}\left(\cos \theta^{\prime}\right) \\
& =P_{l}(\cos \theta) \delta_{l l^{\prime}} .
\end{aligned}
$$

This proves Eq. (A.1).

\section{Appendix B: Integral representations of $j_{l}(u) j_{l}(v)$ and} $j_{l}(u) n_{l}(v)$

In this appendix, we provide two integral representations for the product of two spherical Bessel functions $j_{l}(u) j_{l}(v)$ and $j_{l}(u) n_{l}(v)$ :

$j_{l}(u) j_{l}(v)=\frac{1}{2} \int_{-1}^{1} \mathrm{~d} \cos \theta \frac{\sin w}{w} P_{l}(\cos \theta)$,

where $w=\sqrt{u^{2}+v^{2}-2 u v \cos \theta}$ and $l$ is an integer.

Proof Using the expansion [65]

$\frac{\sin w}{w}=\sum_{l=0}^{\infty}(2 l+1) j_{l}(u) j_{l}(v) P_{l}(\cos \theta)$,

where $u=|\mathbf{u}|$ and $v=|\mathbf{v}|$ with $\theta$ as the angle between $\mathbf{u}$ and $\mathbf{v}$. Multiplying both sides of (B.2) by $P_{l^{\prime}}(\cos \theta)$ and integrating from 0 to $\pi$ give

$$
\begin{aligned}
& \int_{-1}^{1} \mathrm{~d} \cos \theta \frac{\sin w}{w} P_{l^{\prime}}(\cos \theta) \\
& =\sum_{l=0}^{\infty} \int_{-1}^{1} \mathrm{~d} \cos \theta(2 l+1) j_{l}(u) j_{l}(v) \\
& \quad P_{l}(\cos \theta) P_{l^{\prime}}(\cos \theta)=2 j_{l^{\prime}}(u) j_{l^{\prime}}(v) .
\end{aligned}
$$


Here, the orthogonality, $\int_{-1}^{1} \mathrm{~d} \cos \theta P_{l}(\cos \theta) P_{l^{\prime}}(\cos \theta)=$ $2 /(2 l+1) \delta_{l l^{\prime}}$, is used. This proves Eq. (B.1). We have

$j_{l}(u) n_{l}(v)=-\frac{1}{2} \int_{-1}^{1} \mathrm{~d} \cos \theta \frac{\cos w}{w} P_{l}(\cos \theta), \quad(u<v)$,

where $w=\sqrt{u^{2}+v^{2}-2 u v \cos \theta}$.

Proof Using the expansion [65]

$\frac{\cos w}{w}=-\sum_{l=0}^{\infty}(2 l+1) j_{l}(u) n_{l}(v) P_{l}(\cos \theta), u<v$

where $u=|\mathbf{u}|$ and $v=|\mathbf{v}|$ with $\theta$ the angle between $\mathbf{u}$ and $\mathbf{v}$. Multiplying both sides of (B.5) by $P_{l^{\prime}}(\cos \theta)$ and integrating from 0 to $\pi$ give

$$
\begin{aligned}
& \int_{-1}^{1} \mathrm{~d} \cos \theta \frac{\cos w}{w} P_{l^{\prime}}(\cos \theta) \\
& =-\sum_{l=0}^{\infty}(2 l+1) j_{l}(u) n_{l}(v) \\
& \quad \times \int_{-1}^{1} \mathrm{~d} \cos \theta P_{l}(\cos \theta) P_{l^{\prime}}(\cos \theta) \\
& =-\sum_{l=0}^{\infty}(2 l+1) j_{l}(u) n_{l}(v) \frac{2}{2 l+1} \delta_{l l^{\prime}} \\
& =-2 j_{l^{\prime}}(u) n_{l^{\prime}}(v) .
\end{aligned}
$$

This proves Eq. (B.4).

\section{References}

1. D.V. Vassilevich, Phys. Rep. 388(5), 279 (2003)

2. N. Graham, M. Quandt, H. Weigel, Spectral Methods in Quantum Field Theory, vol. 777 (Springer, Berlin, 2009)

3. L.E. Ballentine, Quantum Mechanics: a Modern Development (World Scientific Publishing Company, Singapore, 1998)

4. L. Culumovic, D. McKeon, Phys. Rev. D 38(12), 3831 (1988)

5. F. Dilkes, D. McKeon, Phys. Rev. D 53(8), 4388 (1996)

6. D. McKeon, Mod. Phys. Lett. A 6(40), 3711 (1991)

7. M. Kotani, T. Sunada, Commun. Math. Phys. 209(3), 633 (2000)

8. K. Groh, F. Saueressig, O. Zanusso, arXiv preprint arXiv:1112.4856 (2011)

9. D. Fliegner, M.G. Schmidt, C. Schubert, Zeitschrift für Physik C Particles and Fields 64(1), 111 (1994)

10. A. Barvinsky, G. Vilkovisky, Nucl. Phys. B 282, 163 (1987)

11. A. Barvinsky, G. Vilkovisky, Nucl. Phys. B 333(2), 471 (1990)

12. A. Barvinsky, G. Vilkovisky, Nucl. Phys. B 333(2), 512 (1990)

13. V. Mukhanov, S. Winitzki, Introduction to Quantum Effects in Gravity (Cambridge University Press, Cambridge, 2007)

14. H. Pang, W.S. Dai, M. Xie, Eur. Phys. J. C 72(5), 1 (2012)
15. W.S. Dai, M. Xie, J. High Energy Phys. 2009(02), 033 (2009)

16. W.S. Dai, M. Xie, Phys. Lett. A 311(4), 340 (2003)

17. W.S. Dai, M. Xie, J. Math. Phys. 48(12), 123302 (2007)

18. W. Dai, M. Xie, EPL (Europhys. Lett.) 72(6), 887 (2005)

19. E.B. Davies, Heat Kernels and Spectral Theory, vol. 92 (Cambridge University Press, Cambridge, 1990)

20. A.A. Bytsenko, G. Cognola, V. Moretti, S. Zerbini, E. Elizalde, Analytic Aspects of Quantum Fields (World Scientific, Singapore, 2003)

21. N. Graham, R. Jaffe, V. Khemani, M. Quandt, M. Scandurra, H. Weigel, Nucl. Phys. B 645(1), 49 (2002)

22. E. Farhi, N. Graham, P. Haagensen, R.L. Jaffe, Phys. Lett. B 427(3), 334 (1998)

23. E. Farhi, N. Graham, R. Jaffe, H. Weigel, Nucl. Phys. B 585(1), $443(2000)$

24. E. Farhi, N. Graham, R. Jaffe, H. Weigel, Nucl. Phys. B 630(1), 241 (2002)

25. S.J. Rahi, T. Emig, N. Graham, R.L. Jaffe, M. Kardar, Phys. Rev. D 80(8), 085021 (2009)

26. F.W. Olver, D.W. Lozier, R.F. Boisvert, C.W. Clark, NIST Handbook of Mathematical Functions (Cambridge University Press, Cambridge, 2010)

27. Y.V. Gusev, Nucl. Phys. B 807(3), 566 (2009)

28. C.J. Joachain, Quantum Collision Theory (North-Holland Publishing Company, Amsterdam, 1975)

29. R.I. Nepomechie, Phys. Rev. D 31(12), 3291 (1985)

30. D.M. McAvity, H. Osborn, Class. Q. Grav. 8(4), 603 (1991)

31. T.P. Branson, P.B. Gilkey, K. Kirsten, D.V. Vassilevich, Nucl. Phys. B 563(3), 603 (1999)

32. D.M. McAvity, H. Osborn, Class. Q. Grav. 8(8), 1445 (1991)

33. A. Van de Ven, Nucl. Phys. B 250(1), 593 (1985)

34. I. Avramidi, Theor. Math. Phys. 79(2), 494 (1989)

35. I. Avramidi, Phys. Lett. B 238(1), 92 (1990)

36. I. Avramidi, Nucl. Phys. B 355(3), 712 (1991)

37. D. Fliegner, P. Haberl, M. Schmidt, C. Schubert, Ann. Phys. 264(1), 51 (1998)

38. A.E. Van de Ven, Class. Q. Grav. 15(8), 2311 (1998)

39. A. Barvinsky, Y.V. Gusev, G. Vilkovisky, V. Zhytnikov, J. Math. Phys. 35(7), 3525 (1994)

40. A. Barvinsky, Y.V. Gusev, G. Vilkovisky, V. Zhytnikov, Nucl. Phys. B 439(3), 561 (1995)

41. A. Barvinsky, Y.V. Gusev, G. Vilkovisky, V. Zhytnikov, J. Math. Phys. 35(7), 3543 (1994)

42. A. Codello, O. Zanusso, J. Math. Phys. 54(1), 013513 (2013)

43. G.M. Shore, Nucl. Phys. B 646(1), 281 (2002)

44. V.P. Gusynin, Phys. Lett. B 225(3), 233 (1989)

45. V. Gusynin, Nucl. Phys. B 333(1), 296 (1990)

46. V.P. Gusynin, E. Gorbar, V. Romankov, Nucl. Phys. B 362(1), 449 (1991)

47. W.S. Dai, M. Xie, J. High Energy Phys. 2010(6), 1 (2010)

48. B. Iochum, C. Levy, D. Vassilevich, Commun. Math. Phys. 316(3), 595 (2012)

49. A. Mostafazadeh, Ann. Phys. 341, 77 (2014)

50. J. Mei, Z. Liu, J. Shi, D. Tian, Phys. Rev. B 67(24), 245107 (2003)

51. D. Jena, A.C. Gossard, U.K. Mishra, Appl. Phys. Lett. 76(13), 1707 (2000)

52. M.A. Amin, E.A. Lim, I.S. Yang, Phys. Rev. D 88(10), 105024 (2013)

53. D. Novikov, Phys. Rev. B 76(24), 245435 (2007)

54. A.C. Neto, F. Guinea, N. Peres, K.S. Novoselov, A.K. Geim, Rev. Mod. Phys. 81(1), 109 (2009)

55. P. Arnold, G. Moore, Phys. Rev. Lett. 87(12), 120401 (2001)

56. B. Kastening, Phys. Rev. A 69(4), 043613 (2004)

57. V. Mineev, Phys. Rev. B 69(14), 144429 (2004)

58. V. Mineev, Phys. Rev. B 72(14), 144418 (2005)

59. P.C. Sabatier, J. Math. Phys. 41, 4082 (2000) 
60. L.D. Faddeyev, B. Seckler, J. Math. Phys. 4, 72 (1963)

61. W.M. Liu, B. Wu, Q. Niu, Phys. Rev. Lett. 84(11), 2294 (2000)

62. F. Nicoleau, J. Math. Phys. 41, 5223 (2000)

63. T. Liu, W.D. Li, W.S. Dai, J. High Energy Phys. 2014(6), 1 (2014)
64. S. Flügge, Practical Quantum Mechanics (Springer, New York, 1994)

65. G. Watson, Theory of Bessel Functions, 1922 (Cambridge University Press, Cambridge, 1944) 